\title{
AVALIAÇÃO DO ESTADO NUTRICIONAL DE ESTUDANTES DO ENSINO FUNDAMENTAL NA REDE MUNICIPAL DE CAMPINA GRANDE/PB
}

\author{
EVALUATION OF THE NUTRITIONAL STATUS OF ELEMENTARY \\ SCHOOL STUDENTS IN THE MUNICIPAL NETWORK OF CAMPINA \\ GRANDE / PB
}

\author{
Naryelle da Rocha Silva ${ }^{1}$ \\ Larissa Mirella Araújo de Macêdo ${ }^{2}$ \\ Carla Campos Muniz Medeiros ${ }^{3}$ \\ Geisielly Raquel da Cruz Aguiar ${ }^{4}$ \\ Danielle Franklin de Carvalho ${ }^{5}$ \\ Ana Raquel de Andrade Barbosa Ribeiro ${ }^{6}$
}

RESUMO: OBJETIVO: avaliar o estado nutricional dos estudantes do ensino fundamental da rede municipal de Campina Grande/PB. MÉTODOS: estudo epidemiológico de corte transversal, piloto, através do preenchimento por pesquisadores treinados de um questionário. RESULTADOS: Após a avaliação de 558 adolescentes, desses $51,6 \%$ (288) do sexo masculino, sendo a maioria dos adolescentes eutróficos $66,7 \%$ (372), 20,4\% (114) em estado de sobrepeso, 9,7\% (54) obesos e apenas $3,2 \%$ (18) com baixo peso. Ou seja $30,1 \%$ dos participantes apresentavam excesso de peso, desses, $15,8 \%$ (88) do sexo masculino. Os resultados são corroborados pela literatura que afirma que prevalência de excesso de peso em adolescentes oscila, nos dois sexos, de $16 \%$ a $19 \%$ nas Regiões Nordeste. CONCLUSÃO: O presente trabalho visa contribuir para conhecimento da prevalência de excesso de peso nos adolescentes da cidade de Campina Grande, comparar os dados obtido com a estimativa nacional e poder servir de subsídio para

\footnotetext{
1 Nutricionista; Docente da Faculdade Maurício de Nassau - CG; Mestre em Saúde Pública; Doutoranda em Saúde da Criança e do Adolescente - UFPE.

${ }^{2}$ Médica; Residente em Ginecologia e Obstetrícia - UFCG.

${ }^{3}$ Médica Endocrinologista; Docente da Universidade Estadual da Paraíba - UEPB; Doutora em Saúde da Criança e do Adolescente - FCM-UNICAMP.

${ }^{4}$ Enfermeira; Mestranda em Saúde Pública - UEPB.

5 Farmacêutica-Bioquímica; Docente da Universidade Estadual da Paraíba - UEPB; Doutora em Saúde da Criança e do Adolescente - UFPE.

${ }^{6}$ Médica Endocrinologista; Docente da Universidade Federal de Campina Grande - UFCG; Mestre em Saúde Pública - UEPB.
} 
implantação de estratégias que efetivamente previnam a obesidade, outros fatores de risco, e suas consequências, em estágios precoces da vida.

Palavras chave: Obesidade; Estado Nutricional; Comportamento do Adolescente.

ABSTRACT: OBJECTIVE: to evaluate the nutritional status of elementary school students in the municipal network of Campina Grande / PB. METHOD: epidemiological, cross-sectional, pilot study, through the completion of a questionnaire by trained researchers. RESULTS: After assessing 558 adolescents, $51.6 \%$ (288) of whom were male, with the majority of eutrophic adolescents being $66.7 \%$ (372), 20.4\% (114) in an overweight state, $9.7 \%$ (54) obese and only 3.2\% (18) with low weight. In other words, $30.1 \%$ of the participants were overweight, of these, $15.8 \%$ (88) were male. The results are corroborated by the literature that states that the prevalence of overweight in adolescents ranges, in both sexes, from $16 \%$ to $19 \%$ in the Northeast Regions. CONCLUSION: The present study aims to contribute to the knowledge of the prevalence of overweight among adolescents in the city of Campina Grande, to compare the data obtained with the national estimate and to be able to serve as a subsidy for the implementation of strategies that effectively prevent obesity, other risk factors, and its consequences, at early stages of life.

Keywords: Obesity; Nutritional status; Adolescent Behavior. 


\section{INTRODUÇÃO}

A adolescência, período compreendido ente 10 e 19 anos (WHO,1995), é uma fase da vida caracterizada por processos de crescimento e de desenvolvimento em que ocorrem mudanças biopsicossociais marcantes. Nesse período de transição entre a infância e a fase adulta o comportamento se altera devido à influência de fatores diversos em resposta à busca por uma nova identidade (LOURENÇO; QUEIROZ, 2010).

Diante dessas transformações, nessa fase é comum que eles alterem seus estilos de vida e que adquiram novos hábitos, os quais podem se refletir em suas condições de saúde e em seu comportamento alimentar pelo resto da vida (GARCIA; GAMBARDELLA; FRUTUOSO, 2003). Neste sentido, o aumento da prevalência de sobrepeso e obesidade em idades cada vez mais precoces tem despertado a preocupação de pesquisadores e profissionais da área da saúde, em razão dos danos e agravos à saúde provocados pelo excesso de peso, tais como hipertensão arterial sistêmica, cardiopatias, diabetes mellitus, dislipidemias, dentre outros. (ENES; SLATER, 2010).

Nos países economicamente desenvolvidos, a prevalência de obesidade já atinge níveis expressivos a ponto de a Organização Mundial da Saúde (OMS) considerar a obesidade como um importante problema de Saúde Pública. Nos Estados Unidos da América (EUA), o excesso de peso já ultrapassa $60,0 \%$ dos adultos e apresenta aumento rápido entre crianças e adolescentes, impactando sobre a saúde e a qualidade de vida geral da população. (KOSTI; PANAGIOTAKOS, 2006).

No Brasil, o sobrepeso e a obesidade vêm aumentando em todas as faixas etárias e em ambos os sexos, em todos os níveis de renda ou etnia, sendo a velocidade de crescimento mais expressiva na população com menor rendimento familiar (IBGE, 2010). Em adultos, o excesso de peso e a obesidade atingiram 56,9\% e $20,8 \%$ da população em 2013, respectivamente (IBGE, 2015). 
Em adolescentes, em 2008-2009 o excesso de peso já havia aumentado seis vezes no sexo masculino e quase três vezes no feminino, alcançando a prevalência de cerca de $20,0 \%$ em ambos os sexos. A Pesquisa de Orçamento Familiar (POF) de 2008-2009 já indicava que em menos de 10 anos cerca de dois terços dos adultos brasileiros poderiam ter excesso de peso (IBGE, 2010).

O presente estudo se propõe a estabelecer o atual estado nutricional dos adolescentes do ensino fundamental na rede municipal de Campina Grande/PB.

\section{METODOLOGIA}

Trata-se de um estudo epidemiológico de corte transversal, piloto, que serviu de subsídio e instrumento de treinamento para pesquisadores envolvidos no ensaio clínico randomizado (ECR) "Impacto do exergame na função cardiorrespiratória, macro e microcirculação de adolescentes com excesso de peso: estudo de intervenção randomizado", que teve seus dados coletados no período de setembro de 2017 a março de 2018, respeitando o calendário da rede de ensino.

A pesquisa foi realizada em escolas de grande porte (igual ou superior a 500 alunos) (TENÓRIO et al., 2010) na zona urbana da cidade de Campina Grande, Paraíba com Adolescentes com idade entre 10 a 19 anos, matriculados entre o $5^{\circ} \mathrm{e}$ o $9^{\circ}$ ano do ensino fundamental. Por critério de inclusão, os alunos precisavam: Estar devidamente matriculado na Escola participante; Portar autorização dos pais; Estar presente nas datas de realização da coleta. E de exclusão: Ter alguma limitação motora ou mental que os impedisse de participar da pesquisa.

Foi utilizado como instrumento de coleta de dados um questionário elaborado pelos pesquisadores do Núcleo de Estudos e Pesquisas Epidemiológicas (NEPEAPÊNDICE A) que continha além do espaço para registrar os dados antropométricos aferidos a informação do uso e do tipo de rede social pelos adolescentes (Facebook, Whatsapp, Snapchat, Twitter, Instagram Outras), tendo em vista que a informação relativa ao uso de rede social era relevante para o ensaio clínico a ser realizado posteriormente. 
O Estado nutricional foi avaliado pelo índice de massa corporal (IMC), construído a partir da razão do peso (em $\mathrm{Kg}$ ) pelo quadrado da altura $(\mathrm{em} \mathrm{m})$. O peso foi determinado através de balança digital (Tonelli®), com precisão de $100 \mathrm{~g}$ e a altura através de antropômetro fixo (Seca $\AA)$, com acuidade de um centímetro.

A categorização do estado nutricional foi baseada no escore-z de IMC-Idade para adolescentes de 10 a 19 anos de modo que os pontos de corte estabelecidos estão de acordo com a tabela 1 (WHO,1995).

Tabela 1. Pontos de corte escore-z IMC-Idade.

$\begin{array}{cc}\text { Percentil do IMC } & \text { Diagnóstico Nutricional } \\ <\text { Percentil } 5 & \text { Baixo Peso } \\ \geq \text { Percentil } 5 \text { e }<\text { Percentil 85 } & \text { Adequado ou Eutrófico } \\ \geq \text { Percentil } 85 & \text { Sobrepeso }\end{array}$

Os dados antropométricos foram obtidos em duplicata, sendo utilizada a média dos valores verificados. Ademais, caso entre as aferições no mesmo escolar se observasse diferença além do aceitável, que são: 0,5 centímetros para a altura e 100 gramas para o peso, elas eram novamente realizadas por outro pesquisador, que não conhecia a avaliação anterior.

Os dados colhidos foram avaliados em programa SPSS para Windows, a partir de onde foi realizado o processamento dos mesmos e gerado dados estatísticos para análise do perfil antropométrico dos pacientes estudados.

O estudo foi desenvolvido em conformidade com a Resolução 466/201239 do Conselho Nacional de Saúde e foi aprovado pelo Comitê de Ética em Pesquisa da Universidade Estadual da Paraíba, CAAE: 84019518.3.0000.518. O menor, cujo responsável legal assinou o Termo de Consentimento Livre e Esclarecido (TCLE) e assinou o Termo de Assentimento (TA). 


\section{RESULTADOS}

A amostra inicial foi composta por 564 participantes, sendo 6 participantes excluídos por preenchimento incompleto dos dados no questionário, perfazendo uma amostra final de 558 participantes.

Verificou-se que a população estudada teve distribuição equilibrada entre os sexos, $51,6 \%$ (288) do sexo masculino. A maioria, 84,1\% (469) com idade maior que 12 anos e 53,3\% com turno escolar no período da tarde em relação à manhã e com $86,7 \%$ apresentando acesso às redes sociais.

Tabela 2. Caracterização da amostra.

VARIÁVEIS

\section{SEXO}

\section{Masculino}

Feminino

IDADE

10 a 12 anos

$>12$ anos

TURNO

Manhã

Tarde

\section{TOTAL}

n

288

270

89

469

238

320

\section{$\%$}

51,6

48,4

15,9

84,1

Grande percentual dos adolescentes apresentou estado de eutrofia 66,7\% (372), com prevalência de 20,4\% (114) de sobrepeso, 9,7\% (54) obesidade e apenas 3,2\% (18) com baixo peso, como demonstrado abaixo no gráfico 1. 


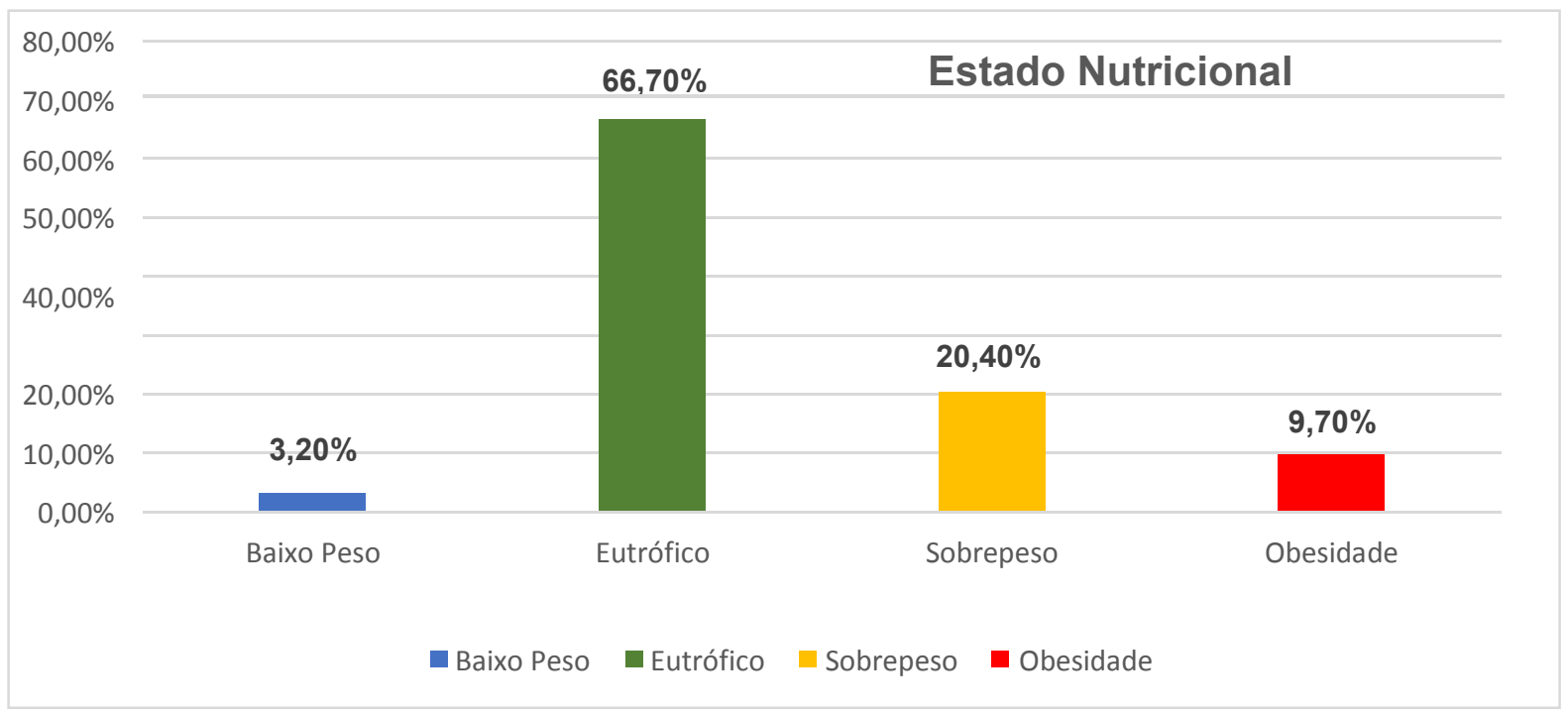

Gráfico 1. Distribuição da amostra segundo estado nutricional.

Ou seja, 30,1\% dos participantes estavam com excesso de peso, desses, $15,8 \%$ (88) eram do sexo masculino e $14,3 \%$ (80) do sexo feminino. O grupo que não apresentou excesso de peso correspondeu a $69,9 \%$ dos participantes e foi composto pelo sexo masculino em $35,8 \%$ (200), enquanto o feminino $34,1 \%$ (190), como observado na tabela 2. Não houve diferença estatisticamente significativa entre a presença de excesso de peso quando se levava o sexo em consideração ( $p$ valor de $0,812)$.

Tabela 3. Distribuição da amostra segundo sexo e estado nutricional.

\begin{tabular}{|c|c|c|c|c|c|c|c|}
\hline VARIÁVEL & \multicolumn{4}{c}{ ESTADO NUTRICIONAL } & \multicolumn{3}{c}{ TOTAL } \\
\hline & \multicolumn{2}{|c|}{ Eutrófico } & \multicolumn{1}{c|}{ Excesso de peso } & & \\
\hline SEXO & $\mathrm{N}$ & $\%$ & $\mathrm{~N}$ & $\%$ & $\mathrm{n}$ & $\%$ \\
\hline Masculino & 88 & 15,8 & 200 & 35,8 & 288 & 51,6 \\
\hline Feminino & 80 & 14,3 & 190 & 34,1 & 270 & 48,4 \\
\hline
\end{tabular}

\section{DISCUSSÃO}

A desnutrição já foi um dos principais problemas na saúde brasileira, mas atualmente apenas $3,4 \%$ da população adolescente, com pouca variação entre os 
sexos, apresenta déficit de peso (IBGE, 2010). Tal dado é compatível com o encontrado no nosso estudo em que $3,2 \%$ dos participantes tinham baixo peso.

Pesquisas de base populacional evidenciam um aumento alarmante das taxas de excesso de peso na adolescência. A comparação dos dados do Estudo Nacional de Despesa Familiar (Endef), realizado em 1974 e 1975, com os dados da Pesquisa sobre Padrões de Vida (PPV), realizada entre 1996 e 1997 (somente nas regiões Sudeste e Nordeste), indica um aumento na prevalência de sobrepeso e obesidade de $4,1 \%$ para $13,9 \%$ em crianças e adolescentes de seis a 18 anos. Apresentando, no Brasil, o aumento percentual anual na prevalência de excesso de peso nas áreas urbanas mais que o dobro do que nas áreas rurais (WANG; MONTEIRO; POPKIN, 2002).

Nos dois sexos a prevalência de excesso de peso no estudo ERICA em 2016 oscilou de $16 \%$ a $19 \%$ nas Regiões Norte e Nordeste (cerca de cinco vezes a prevalência do déficit de peso) e de $20 \%$ a $27 \%$ nas Regiões Sudeste, Sul e CentroOeste (cerca de sete a dez vezes a prevalência do déficit de peso) (BLOCH; CARDOSO; SICHIERI, 2016).

Nesse trabalho, excesso de peso também tendeu a ser mais frequente no meio urbano do que no meio rural, em particular nas Regiões Norte e Nordeste (BLOCH; CARDOSO; SICHIERI, 2016). Em nosso estudo, realizado no meio urbano de Campina Grande/PB, a prevalência de excesso de peso encontrada no sexo masculino foi de $15,8 \%$ sendo bem próxima ao sexo feminino de $14,3 \%$ e compatível com a literatura.

Campos, Leite e Almeida (2006) relataram em Fortaleza/CE a prevalência de sobrepeso/obesidade nas classes de nível socioeconômico mais elevado de $24,8 \%$ e de $17,4 \%$ nas de menor nível. Tal divergência de prevalência de excesso de peso entre classes socioeconômicas também já foi observado em Campina Grande por Nunes, Figueiroa e Alves (2007) quando foram analisados 588 alunos, sendo 292 da escola pública pertencentes às classes econômicas C, D e E, e 296 da escola privada oriundos das classes econômicas $\mathrm{A}$ e $\mathrm{B}$ e foi observado que a proporção de adolescentes com sobrepeso / obesidade também foi significativamente maior nas classes econômicas mais altas (NUNES; FIGUEIROA; ALVES, 2007). 
Ainda segundo Campos, Leite e Almeida (2006) nos estratos sociais de maior nível socioeconômico, o sexo masculino apresentou maior prevalência de sobrepeso/obesidade em relação ao feminino, e nos de menor nível não foi encontrada diferença estatisticamente significante entre os sexos.

O presente estudo também não conseguiu associar o estado nutricional com o sexo nos adolescentes, possivelmente por serem alunos da rede pública e apresentarem menor nível socioeconômico, como relatado.

Logo, o presente trabalho permite observar a tendência de aumento de sobrepeso e obesidade com brusca redução dos índices de baixo peso na cidade de Campina Grande/PB, sendo, portanto, correspondente com a transição epidemiológica nutricional nacional (inversão de perfil nutricional que pode acarretar aumento da prevalência de doenças crônicas no futuro) relatada pela literatura (BERGAMASCHI; ADAMI, 2015).

No mundo essa tendência também se mantém, em uma pesquisa realizada com 130 milhões de jovens com mais de cinco anos de idade (31,5 milhões entre cinco e 19 anos e 97,4 milhões com mais de 20 anos) foi visualizado que enquanto no ano de 1975 as taxas de obesidade nas crianças e adolescentes do mundo aumentavam menos de 1\% (equivalente a cinco milhões de meninas e seis milhões de meninos), em 2016 o índice subiu para quase 6\% em meninas (50 milhões) e quase $8 \%$ em meninos (74 milhões). Combinados, o número de obesos de cinco a 19 anos aumentou mais do que dez vezes no mundo, de 11 milhões em 1975, para 124 milhões em 2016 (ABARCA-GÓMEZ et al., 2017).

Portanto, o excesso de peso vem se tornando um problema emergente no mundo e em Campina Grande/PB isso não é diferente. Para enfrentá-lo, é importante considerar os estágios iniciais de seu desenvolvimento, o que inclui a fase da adolescência.

Nessa perspectiva, informações atualizadas sobre níveis e tendências da estratificação de peso, como apresentado, surgem como ferramentas importantes para quantificar os efeitos na saúde e qualificar o progresso de intervenções neste meio.

Além disso, a avaliação do estado nutricional é fundamental para o acompanhamento adequado do desenvolvimento dos indivíduos, principalmente no 
âmbito escolar, um ambiente propício para atividades educativas com capacidade de encorajamento na promoção de hábitos de vida saudáveis.

$E$, como observado, as intervenções nos hábitos de vida devem ser iniciadas o mais precocemente possível, já que na adolescência ocorrem mudanças importantes na personalidade do indivíduo e por isso é considerada uma fase favorável para a consolidação de hábitos que poderão trazer implicações diretas para a saúde na vida adulta (ENES; SLATER, 2010).

\section{CONCLUSÃO}

O presente trabalho demonstra uma prevalência importante de excesso de peso entre os adolescentes que frequentam a rede municipal de ensino de Campina Grande/PB, em consonância com dados já publicados em outras regiões, conforme já mencionamos. Dessa forma, esse pode servir de subsídio para implantação de estratégias que efetivamente previnam a obesidade e outros fatores de risco, e suas consequências, em estágios precoces da vida.

Tendo em vista que se trata apenas de um estudo piloto, cujo objetivo era estimar a prevalência do excesso de peso na população a ser estudada no estudo de intervenção e também servir como treinamento aos pesquisadores envolvidos, o presente estudo não compara o perfil nutricional dos adolescentes com o nível socioeconômico, assim como também não correlaciona o IMC com a medida da circunferência da cintura não permitindo, portanto, diferenciar se o excesso de peso é proveniente de maior massa de gordura, massa magra, massa óssea ou água corporal e, além disso, não possibilita localizar a presença de adiposidade central, importante fator de risco DCNT. 


\section{REFERÊNCIAS BIBLIOGRÁFICAS}

ABARCA-GÓMEZ, Leandra et al. Worldwide trends in body-mass index, underweight, overweight, and obesity from 1975 to 2016 : a pooled analysis of 2416 population-based

measurement studies in 128.9 million children, adolescents, and adults. The Lancet, [s.I.], v. 390, n. 10113, p. 2627-2642, dez. 2017. Elsevier BV.

ASSOCIAÇÃO BRASILEIRA PARA O ESTUDO DA OBESIDADE E DA SÍNDROME METABÓLICA. Diretrizes brasileiras de obesidade. 4. ed. São Paulo: Abeso, 2016. 188 p.

BAHIA, Luciana; ARAÚJO, Denizar Vianna. Impacto econômico da obesidade no Brasil. Revista Hospital Universitário Pedro Ernesto, [s.l.], v. 13, n. 1, p.13-17, 17 mar. 2014. Universidade de Estado do Rio de Janeiro.

BERGAMASCHI, Daiane; ADAMI, Fernanda Scherer. PERFIL ANTROPOMÉTRICO DE CRIANÇAS E ADOLESCENTES. Revista Ciência e Saúde, São Luís, v. 1, p.53-60, jun. 2015.

BLOCH, Katia Vergetti; CARDOSO, Marly Augusto; SICHIERI, Rosely. Study of Cardiovascular Risk Factors in Adolescents (ERICA): results and potentiality. Revista de Saúde Pública, [s.I.], v. 50 , n. 1, p.1-2, 2016. FapUNIFESP (SciELO).

CAMPOS, Lício de Albuquerque; LEITE, Álvaro Jorge Madeiro; ALMEIDA, Paulo César de. Nível socioeconômico e sua influência sobre a prevalência de sobrepeso e obesidade em escolares adolescentes do município de Fortaleza. Revista de Nutrição, [s.I.], v. 19, n. 5, p.531- 538, out. 2006. FapUNIFESP (SciELO).

ENES, Carla Cristina; SLATER, Betzabeth. Obesidade na adolescência e seus principais fatores determinantes. Revista Brasileira de Epidemiologia, São Paulo, v. 13, p.163-171, fev. 2010.

FACINA, Vanessa Barbosa; BRAGA, Jordana Grazielle Lopes. PERFIL ANTROPOMÉTRICO DE ADOLESCENTES DO RECÔNCAVO DA BAHIA. Revista Ciência em Extensão, [s.i.], v. 9, n. 3, p.21-23, abr. 2013.

GARCIA, Giorgia Christina Barbosa; GAMBARDELLA, Ana Maria Dianezi; FRUTUOSO, Maria Fernanda Petrole. Estado nutricional e consumo alimentar de adolescentes de um centro de juventude da cidade de São Paulo. Revista de Nutrição, [s.I.], v. 16, n. 1, p.41-50, jan. 2003. FapUNIFESP (SciELO).

IBGE, Instituto Brasileiro de Geografia e Estatística. Pesquisa Nacional de Saúde: Brasil e grandes regiões. Rio de Janeiro: Instituto Brasileiro de Geografia e Estatística, 2015. 105 p.

IBGE, Instituto Brasileiro de Geografia e Estatística. Pesquisa de Orçamentos Familiares 2008-2009: Antropometria e estado nutricional de crianças, adolescentes e adultos no Brasil. Rio de Janeiro: lbge, 2010.

KOSTI, Rena I.; PANAGIOTAKOS, Demosthenes B.. The Epidemic of Obesity in Children and Adolescents in the World. Central European Journal Of Public Health, [s.I.], v. 14, n. 4, p.151159, 1 dez. 2006. National Institute of Public Health.

LOURENÇO, Benito; QUEIROZ, Lígia Bruni. Crescimento e desenvolvimento puberal na adolescência. Revista de Medicina, [s.I.], v. 89, n. 2, p.70-75, 19 jun. 2010. Universidade de Sao Paulo Sistema Integrado de Bibliotecas - SIBiUSP. 
NG, Marie et al. Global, regional, and national prevalence of overweight and obesity in children and adults during 1980-2013: a systematic analysis for the Global Burden of Disease Study 2013. The Lancet, [s.I.], v. 384, n. 9945, p.766-781, ago. 2014. Elsevier BV.

NUNES, Marília Medeiros de Araújo; FIGUEIROA, José Natal; ALVES, João Guilherme Bezerra. Excesso de peso, atividade física e hábitos alimentares entre adolescentes de diferentes classes econômicas em Campina Grande (PB). Revista da Associação Médica Brasileira, [s.l.], v. 53, n. 2, p.130-134, abr. 2007. Elsevier BV.

RICARDO, Gabriela Dalsasso; GABRIEL, Cristine Garcia; CORSO, Arlete Catarina Tittoni. Perfil antropométrico e adiposidade abdominal de escolares entre 6 a 10 anos de idade do Sul do Brasil. Revista Brasileira de Cineantropometria e Desempenho Humano, [s.I.], v. 14, n. 6 , p.636-643, 16 nov. 2012. Universidade Federal de Santa Catarina (UFSC).

SILVEIRA, Adriana Márcia. Crianças e adolescentes com excesso de peso: repercussões do atendimento multidisciplinar sobre a mudança de hábitos alimentares, medidas antropométricas e parâmetros bioquímicos. 2009. 106 f. Dissertação (Mestrado) - Curso de Medicina, Ciências da Saúde, Faculdade de Medicina da Ufmg, Belo Horizonte, 2009.

SOUZA, Amanda de Moura et al. ERICA: intake of macro and micronutrients of Brazilian adolescents. Revista de Saúde Pública, [s.I.], v. 50, n. 1, p.1-15, 2016. FapUNIFESP (SciELO).

SUS, Departamento Nacional de Auditoria do. PREVEnÇÃO E TRATAMENTO. 2013.

TENÓRIO, Maria Cecília Marinho et al. Atividade física e comportamento sedentário em adolescentes estudantes do ensino médio. Revista Brasileira de Epidemiologia, [s.I.], v. 13, n. 1, p.105-117, mar. 2010. FapUNIFESP (SciELO).

WANG, Youfa; MONTEIRO, Carlos; POPKIN, Barry M. Trends of obesity and underweight in older children and adolescents in the United States, Brazil, China, and Russia. The American Journal Of Clinical Nutrition, [s.I.], v. 75, n. 6, p.971-977, 1 jun. 2002. Oxford University Press (OUP).

WHO, World Health Organization. Phisical Status: the use and interpretation of anthropometry. Geneva: World Health Organization, 1995. 463 p. (854). 